Article

\title{
Quantifying Cutting and Wearing Behaviors of TiN- and CrN- Coated AISI 1070 Steel
}

\author{
Ahmet Cakan ${ }^{1, *}$, Vedat Ozkaner ${ }^{2}$ and Mustafa M. Yildirim ${ }^{3}$ \\ ${ }^{1}$ Department of Mechanical Engineering, Abant Izzet Baysal University, Bolu, Turkey \\ ${ }^{2}$ Department of Electirical-Electronic Engineering, Mustafa Kemal University, Hatay, Turkey \\ ${ }^{3}$ Faculty of Technical Education, Department of Metallurgy, University of Firat, Elazig, Turkey \\ E-mails: ahcakan@gmail.com (A.C.); vozkaner@gmail.com (V.O.); mmyildirimprof@gmail.com \\ (M. M. Y.) \\ * Author to whom correspondence should be addressed.
}

Received: 18 September 2008; in revised form: 6 October 2008 / Accepted: 31 October 2008 / Published: 5 November 2008

\begin{abstract}
Hard coatings such as titanium nitride (TiN) and chromium nitride $(\mathrm{CrN})$ are widely used in cutting and forming tools against wear and corrosion. In the present study, hard coating films were deposited onto AISI 1070 steels by a cathodic arc evaporation plating (CAVP) technique. These samples were subjected to wear in a conventional lathe for investigating the tribological behaviour of coating structure, and prenitrided subsurface composition was characterized using scanning electron microscopy (SEM), line scan analyses and X-ray diffraction (XRD). The wear properties of TiN- and CrNcoated samples were determined using an on-line monitoring system. The results show that TiN-coated samples demonstrate higher wear resistance than $\mathrm{CrN}$-coated samples.
\end{abstract}

Keywords: TiN-coating, CrN-coating, cutting performance, tool wear on-line monitoring, cutting force.

\section{Introduction}

Thin film coating is applied to improve the wear resistance of substrates owing to its hardness and high mechanical strength. Applications of thin films are steadily increasing in various industrial fields [1]. Among many hard coatings, PVD (physical vapor deposition) TiN (titanium nitride), TiC 
(titanium carbide) and $\mathrm{CrN}$ (chromium nitride) are the most widely used ceramic coatings in cutting tools due to their high hardness and wear resistance, low coefficient of friction [2], high temperature strength, chemical stability [3], and corrosion resistance [4]. However, application of PVD hard coatings to the substrate materials cannot guarantee the optimal tribological performance without pretreatment of the substrate materials due to plastic deformation of the substrate, which results in eventual coating failure. The surface treatment provides hardening and increases the load support effect for the substrate [5].

Many approaches aim to enhance the load bearing capacity of the coated surfaces and improve the adhesion of the coatings such as deposition of an interlayer material or compound prior to the coating or the treatment of the surface by nitriding [6-9], carburizing, borizing, nitrocarburizing and so on. However, in terms of the cost and complexity, nitriding or carburising is a better candidate than the other methods. Previous research showed the beneficial effects of nitriding steel before PVD coating. For example, nitriding was found to increase the lifetime of cutting tools made of tool steel [10].

The aim of this study is to investigate the possibility of using PVD hard coatings such as TiN and $\mathrm{CrN}$ on the medium carbon substrates, which are typically used in cutting tool applications. Therefore, TiN and CrN hard coatings were deposited on prenitrided medium carbon steel (AISI 1070). The steel substrates were nitrided in molten salt bath before the coating process. On-line monitoring system was used to investigate the wear resistance of $\mathrm{CrN}$ and TiN coatings, which were applied on AISI 1070 tool steel using the cathodic arc evaporation process technique. Experiments were carried out in a conventional lathe and the following parameters have been measured: the wear volume, cutting force, feed force, and thrust force of the counter material. The performance of the prenitrided TiN- and CrNcoated cutting tools was comparatively studied.

\section{Experimental Procedure}

\subsection{Sample Preparation}

Substrate samples with the size of $12 \mathrm{mmx} 12 \mathrm{mmx} 100 \mathrm{~mm}$ were cut from medium carbon steel AISI 1070. The samples were heat-treated in a furnace at a temperature of $760{ }^{\circ} \mathrm{C}$ for a period of 20 minutes and then they were quenched starting from the tip until the room temperature. The final hardness of the tool samples was about $62 \mathrm{HRC}$. All the samples were tempered at $180^{\circ} \mathrm{C}$ for a period of $40 \mathrm{~min}$ with the following composition (wt\%) $0.794 \% \mathrm{C}, 0.0229 \% \mathrm{Si}, 0.023 \% \mathrm{~S}, 0.016 \% \mathrm{P}, 0.3487 \% \mathrm{Mn}, 0.0396 \%$ $\mathrm{Ni}, 0.0133 \% \mathrm{Cr}, 0.0016 \mathrm{Mo}, 0.0012 \% \mathrm{~V}, 0.02 \% \mathrm{Cu}, 0.0016 \% \mathrm{Ti}, 0.0011 \% \mathrm{Sn}$, and $0.0273 \% \mathrm{Al}$. These samples were subsequently grounded, hardened, nitrided, polished and cleaned before the coating. The steps for the sample preparation are given in Fig.1.

\subsection{Salt Bath Nitriding}

To obtain a nitrided layer, nitriding was used as the thermo-chemical surface treatment method, which improves the wear resistance of cutting tools. The cutting tools made of steel grade AISI 1070 were nitrided in a molten salt bath $(\mathrm{CN})$ at about $570^{\circ} \mathrm{C}$ for $2 \mathrm{~h}$. It is reported that, in a conventional process with high nitriding potential, partially compound zone of about $30 \mu \mathrm{m}$ thick was formed [11]. 
The inner part is reported to be a $\gamma^{\prime}-\mathrm{Fe}_{4} \mathrm{~N}, \varepsilon-\mathrm{Fe}_{2-3}(\mathrm{NC})$, or mixed $\varepsilon-\gamma^{\prime}$ phase [11]. Conventional molten salt bath can be used to achieve the above described structure.

\subsection{Coating}

TiN and $\mathrm{CrN}$ coatings were deposited onto prenitrided cutting tools by using cathodic arc evaporation technique (CAVP) as the PVD coating method. Prior to coating, the cutting tool samples were polished by mechanical methods to the surface roughness of $\mathrm{R}_{\mathrm{a}} \sim 0.1 \mu \mathrm{m}$. Then these samples were cleaned in an ultrasonic bath using basic alkali cleaner. The samples were vertically fixtured on a continuously rotating planetary holder inside the vacuum chamber. The purity of $\mathrm{N}_{2}$ gas used was 99.998\% and that of the Ti target was $99.6 \%$. Prior to deposition, chamber was further cleaned by glow discharge by using high purity $\mathrm{N}_{2}$ gas with a partial pressure of 15-20 mtorr and $-1000 \mathrm{~V}$ substrate bias voltage. During TiN deposition, the chamber pressure was maintained at $3 \times 10^{-3}$ torr by introducing nitrogen. A negative bias voltage of $-200 \mathrm{~V}$ was applied to the substrate during deposition. During CrN deposition, the chamber pressure was maintained at $7 \times 10^{-3}$ torr, while a negative bias voltage of $100 \mathrm{~V}$ was applied to the substrate. Deposition period of TiN and $\mathrm{CrN}$ was $45 \mathrm{~min}$ and 30 min, respectively. The thickness of TiN film reached was approximately $1.3 \mu \mathrm{m}$, while that of $\mathrm{CrN}$ film was approximately $3 \mu \mathrm{m}$.

\subsection{Cutting Condition}

Cutting tests were carried out on a $1.2 \mathrm{~kW}$ conventional lathe machine under dry cutting conditions. The tools were tested at the spindle speeds of 190, 240 and $320 \mathrm{rpm}$ (at cutting speeds of $23.89 \mathrm{~m} \mathrm{~min}^{-}$ 1, $30.18 \mathrm{~m} \mathrm{~min}^{-1}$ and $41.12 \mathrm{~m} \mathrm{~min}^{-1}$ ) with a feed rate $0.081 \mathrm{~mm} \mathrm{rev}^{-1}$. A depth of cut of $0.8 \mathrm{~mm}$ was used for shape manufacturing and was kept constant throughout the tests.

\subsection{Workpiece Materials}

The cutting performance tests were performed on DIN 9SMnPb36 steel bars. Based on the carbon steel standard of AISI-SAE, its composition is (wt $\%$ ) $0.15 \% \mathrm{C}, 0.05 \% \mathrm{Si}, 1.50 \% \mathrm{Mn}, 0.100 \% \mathrm{P}$, $0.37 \% \mathrm{~S}$, and $0.15 \% \mathrm{~Pb}$. The workpiece material used has a dimension of $200 \mathrm{~mm}$ of length and 40 $\mathrm{mm}$ of diameter.

\subsection{On-line Monitoring of Tool Wear}

Many systems were developed for indirectly detecting tool wear in turning and milling operations [12-16]. In the present study, the amount of flank wear on a turning tool is indirectly determined, without interrupting the machining operation, by monitoring changes in the workpiece diameter using a photo electronic sensor [17-19]. The sensor consists of a bifurcated optical fibre, a laser diode (650 $\mathrm{nm} 20 \mathrm{~mW}$ Visible red) as the light source and a photodiode (IPL10530DAL) as the detector, which has amplification circuit. The flow chart of the experimental process is shown in Fig. 1.

A schematic diagram of the experimental set-up is shown in Fig. 2. The optical fiber is held in front of the workpiece diametrically opposite to the cutting tool. The optical fiber is fixed in such a way that it lags the cutting tip by a certain distance and its axis intersects the axis of rotation of the workpiece. 
This is to ensure that the laser beam is incident on and reflected back by the freshly produced work surface. The optical fiber used has a core of $3 \mathrm{~mm}$ diameter. The core fibers are coupled with brass sleeves at the ends. A $20 \mathrm{~mW}$ Laser diode is used as the light source. The power of the laser beam should be such that it does not drive the photodiode into the saturation region and yet provides signals of sufficient amplitude. It is necessary to ensure that the laser beam produced is of a constant intensity throughout the experiments. A brass coupling is used to connect the source fibers of the sensor to the laser diode. This is to ensure that there is no relative movement between the source fibers and the laser diode.

Figure 1. Experimental process.

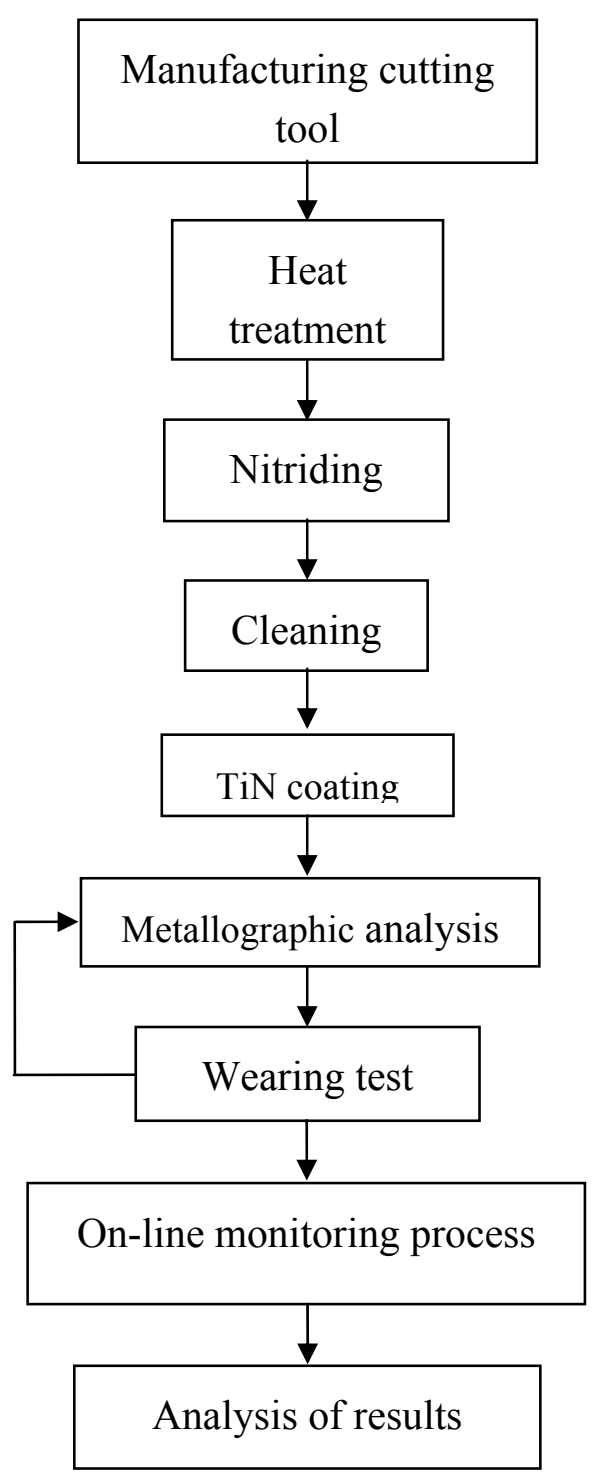

The sensing fibers are connected to the photodiode using another brass coupling to avoid any relative movement between them. This set-up ensures that any change in the output signal is only because of the change in the intensity of the reflected light. The cable carrying the output signal from the photodiode is connected to a data logger. The data were recorded every second by the data logger and were transferred to a PC by using serial port. 
Figure 2. Schematic diagram of the experimental set-up.

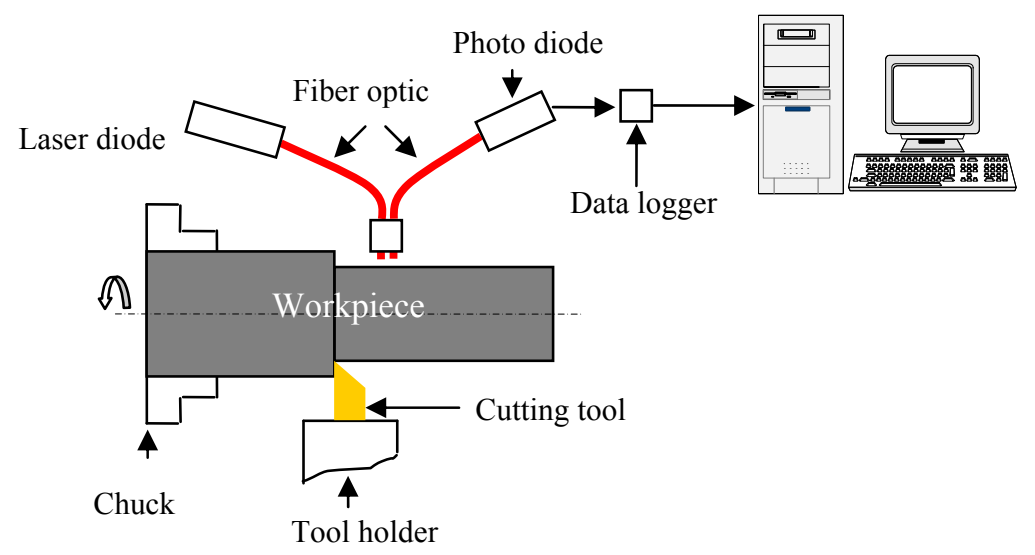

\subsection{The Cutting Force Measurements}

When a cylindrical workpiece is turned, the cutting force $\mathbf{F}$, as shown in Eq. (1), may be divided into three vector force components (see Fig. 3 for vector orientation):

Figure 3. Vector orientation.

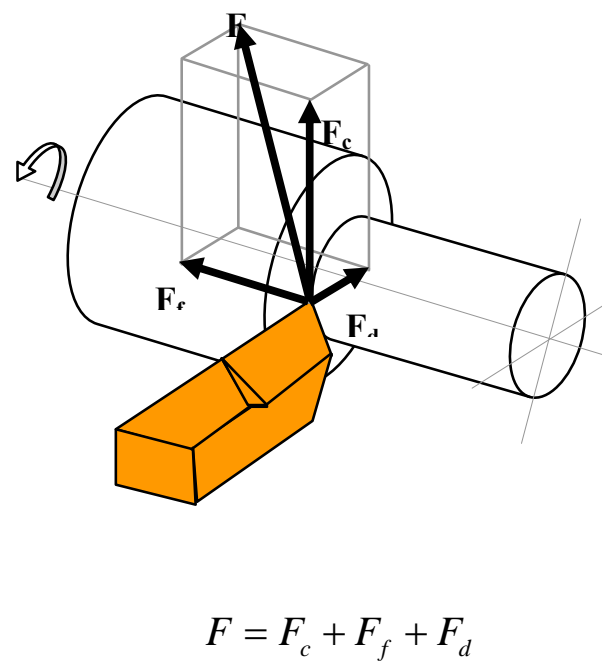

Where $\boldsymbol{F}_{c}$ is the tangential component, $\boldsymbol{F}_{f}$ is the feed force component and $\boldsymbol{F}_{d}$ is the radial force. Force measurement is essential to understanding the cutting mechanism such as the effect of cutting variables on the cutting force, the machinability of the work piece, the process of chip formation and tool wear. The need for measuring the cutting forces is due to the fact that they change with the progress of tool wear. Therefore, tool wear can be determined by monitoring the force variation. The cutting force measurement is a good indicator in detecting tool wear. For this purpose, many dynamometers have been developed [20-22].

During the experiments, these three forces on the cutting tool were measured. These orthogonal components of the force were measured by using the strain gauge based on a three component-cutting force dynamometer [20]. The output signals obtained from the dynamometer for the forces were 
amplified by using three strain gauge amplifiers. The cables carrying the output signal from these amplifiers were connected to the data logger. The data were logged at $1 \mathrm{~s}$ interval and were transferred to the PC once the measurement was complete.

\section{Results and Discussion}

Turning experiments were performed with prenitrided medium carbon steel cutting tools coated with $\mathrm{CrN}$ and TiN. CrN- and TiN-coated tools were tested under the same cutting conditions. The geometrical configurations conform to Turkish Standard (TS 9044). The material of workpiece was $\mathrm{DIN} 9 \mathrm{SMnPb} 36$ steel. The dimensions of the cylindrical workpiece were $40 \mathrm{~mm}$ in diameter and 200 $\mathrm{mm}$ in length. The cutting parameters of the lathe were as follows: feeding rate was $0.081 \mathrm{~mm} \mathrm{rev}^{-1}$, spindle speeds were 190, 240 and $320 \mathrm{rpm}$ (cutting speeds were $23.89 \mathrm{~m} \mathrm{~min}^{-1}, 30.18 \mathrm{~m} \mathrm{~min}^{-1}$, and $41.12 \mathrm{~m} \mathrm{~min}^{-1}$ ), and depth of cut was $0.8 \mathrm{~mm}$. The total length of cut was $150 \mathrm{~mm}$. No cutting fluid was added throughout the turning experiment.

The workpiece was held in a three-jaw chuck and supported in the centre at its tailstock. The other cutting parameters were set as required for the particular experiments. After machining with a fresh tool for about $15 \mathrm{~mm}$ length on the workpieces, the process was stopped and initial gap between the sensor and the workpiece was adjusted to $2.3 \mathrm{~mm}$. The machining operation was then continued for the rest of the workpiece. During machining, the sensor output was continuously recorded. The metal cutting performance of coated tools is a function of substrate, coating, and geometry of the cutting edge. For the given metal cutting test, we kept the substrate and the geometry constant. Therefore, differences in the tool wear and cutting forces observed during the test should be attributed only to the differences in the properties of the coated materials and their adhesion to the substrate. The results are summarized in Fig 4.

\subsection{Voltage Variation}

In most of the experiments, an initial increase in the voltage was observed in contrary to an expected decrease. This phenomenon was also observed and reported by other studies [17,18,23]. The amount of light reflected from the workpiece surface and detected by the photodiode is a function of two parameters: firstly, the distance between the sensor and the workpiece surface, and secondly reflectance of the workpiece. A small nose radius was formed at the sharp cutting tip of the tool due to flank wear sometime after the machining operation was started with a new tool. This leads to a better surface quality of the workpiece and thus a higher reflectance. The gap between the workpieces and the sensor reduces due to the flank wear and notching on the cutting tool, which leads to further drop in the voltage signal during the machining operation. This phenomenon was also observed and reported elsewhere [17].

\subsection{Wear}

Turning tests have been conducted in order to study the wear mechanism of the $\mathrm{CrN}$ and TiN coatings during the cutting process. The relationship between the flank wear and the cutting length of the $\mathrm{CrN}$ - and TiN-coated cutting tools is shown in Fig. 4. Our results indicated that the TiN coating 
had a better wear resistance than $\mathrm{CrN}$ coating. The turning tests conducted at higher cutting speeds showed that the flank wear increased with the increasing cutting speed. Also in higher speeds, TiNcoated cutting tool had a higher wear resistance than CrN-coated one as can be seen in Fig. 4 .

Figure 4. Comparison of wear properties at different rotational speeds at a feed speed of $0.081 \mathrm{~mm} \mathrm{rev}^{-1}$ for (a) TiN-coated and (b) CrN-coated cutting tools.

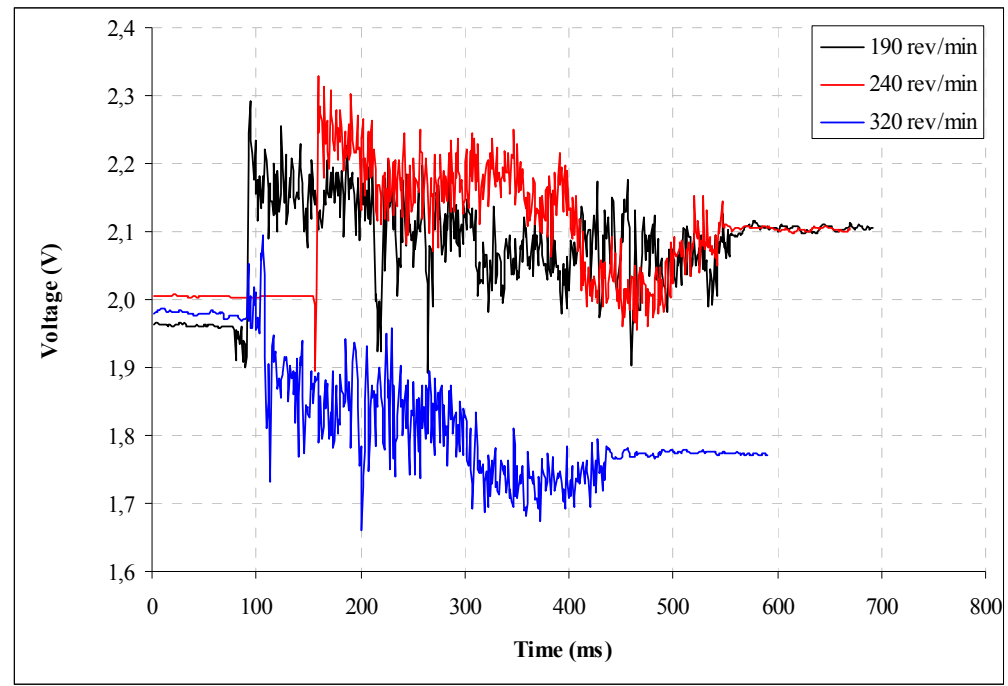

(a)

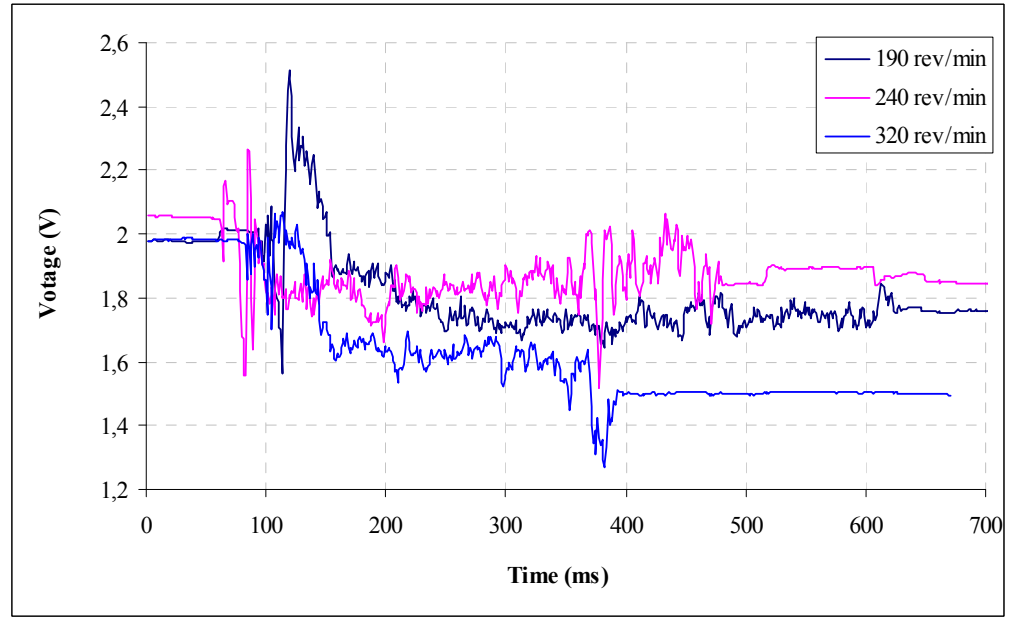

(b)

\subsection{Adhesion}

As the TiN and $\mathrm{CrN}$-coated tools advance, the turning workpiece material continues to adhere to the cutting edge and flank face. Wear progresses as the adhered layers, TiN and $\mathrm{CrN}$ coatings and even the prenitrided high carbon steel substrate are carried by the flowing chips. The wear phenomenon taking place in TiN- and CrN-coated prenitrided high carbon steel after $150 \mathrm{~mm}$ of cutting length appears in Fig.5. One can see a large amount of adhered layer, which is the transferred DIN 9SMnPb36 steel material, covered in the flank face. As the chromium content of the coating material increases, the 
adhesion phenomenon in the dry turning becomes more accentuated [24, 25]. Therefore, the CrNcoated tool had a larger flank wear than TiN-coated one.

Figure 5. Flank wear of the coated samples $\left(\mathrm{V}_{\mathrm{c}}=41.12 \mathrm{~m} \mathrm{~min}^{-1}\right)$ : (a) $\mathrm{CrN}$ and (b) TiN.

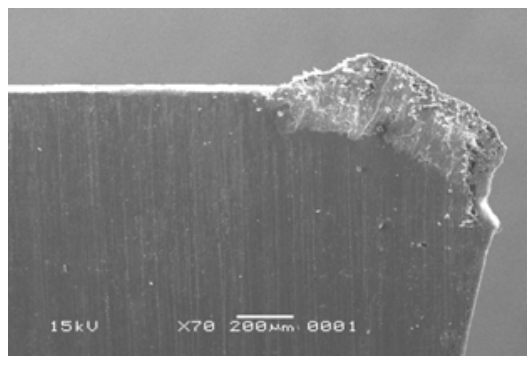

(a)

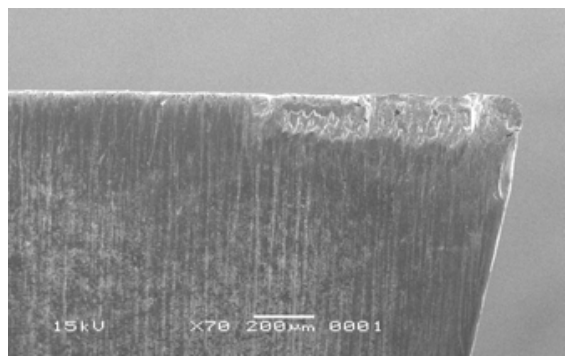

(b)

Our results indicated that the $\mathrm{CrN}$ coatings had strong chemical affinity to the DIN 9SMnPb36 steel during the turning process. According to the phase diagrams, $\mathrm{Fe}$ and $\mathrm{Cr}$ have completely unlimited mutual solid solubility with each other over a temperature of $800{ }^{\circ} \mathrm{C}$ [26]. This was the reason for the adhesion. In the case of the CrN-coated insert, a strong bonding force between the adhered DIN 9SMnPb36 steel material and the $\mathrm{CrN}$ coating was formed, due to high chemical affinity between $\mathrm{Fe}$ and $\mathrm{Cr}$, which occurred at the temperature induced during turning. As the size of the adhered DIN 9SMnPb36 steel material reached a critical value, it was detached, and thus, so were the underlying $\mathrm{CrN}$ coating and small pieces of prenitrided high carbon steel substrate. Thus, the prenitrided high carbon steel substrate was severely fractured by the adhesion. Fig. 5(a) is the SEM micrograph of the wear on the $\mathrm{CrN}$-coated prenitrided medium carbon steel after $150 \mathrm{~mm}$ cutting length of the DIN $9 \mathrm{SMnPb} 36$ steel material. The worn surface exhibits very smooth topography and good conformity with the chip flow pattern. Such smoothly worn surface topography is characteristic of abrasive wear. It is inferred in the present article that the $\mathrm{CrN}$ and $\mathrm{TiN}$ layers are worn off by abrasion process.

\subsection{Cutting Force}

The cutting, feed and radial forces versus cutting speed relationships for the experimental tests are shown in Figs. 6 and 7. Figures also show the effect of different coatings and cutting speeds. For the $\mathrm{CrN}$-coated cutting tools, the cutting force increased when the cutting speed was increased from low to medium.

The force, however, decreased when the cutting speed was at the maximum level. The feed and radial forces were about twice lower than the cutting force. Both other forces, however, decreased with and increase in cutting speed. For the TiN-coated cutting tools, all the forces decreased with an increase in cutting speed. The correlations between the voltage $(\mathrm{mV})$ and force $(\mathrm{kN})$ for the variables of cutting, feed and radial forces were derived from these best-fit equations as shown in Figs. 6 and 7. 
Figure 6. Comparison of cutting forces at different rotational speeds at a feed speed of $0.081 \mathrm{~mm} \mathrm{rev}^{-1}$ for TiN-coated cutting tools: (a) cutting force: $y=-0.501 x+1.0698\left(R^{2}\right.$ $=0.9998 ; P<0.001)$, (b) feed force: $y=80.26 x+124.9\left(R^{2}=0.9995 ; P<0.001\right)$ and (c) radial force: $y=7.94 x+128.5\left(R^{2}=0.9993\right.$; $\left.P<0.001\right) . y$ and $x$ values are in $\mathrm{kN}$ and in $\mathrm{mV}$, respectively.

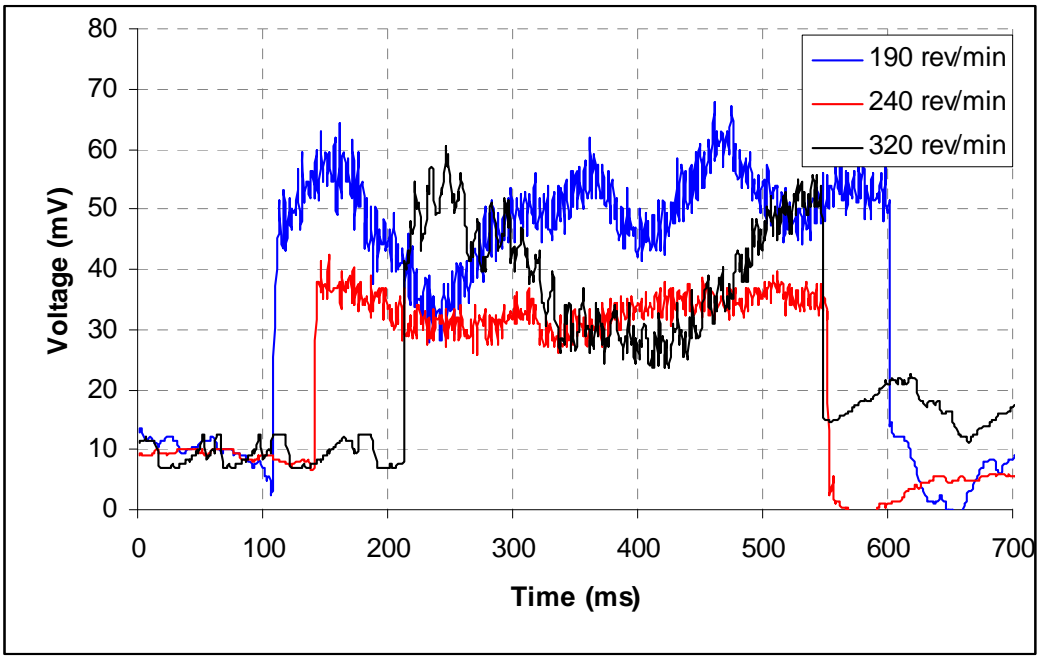

(a)

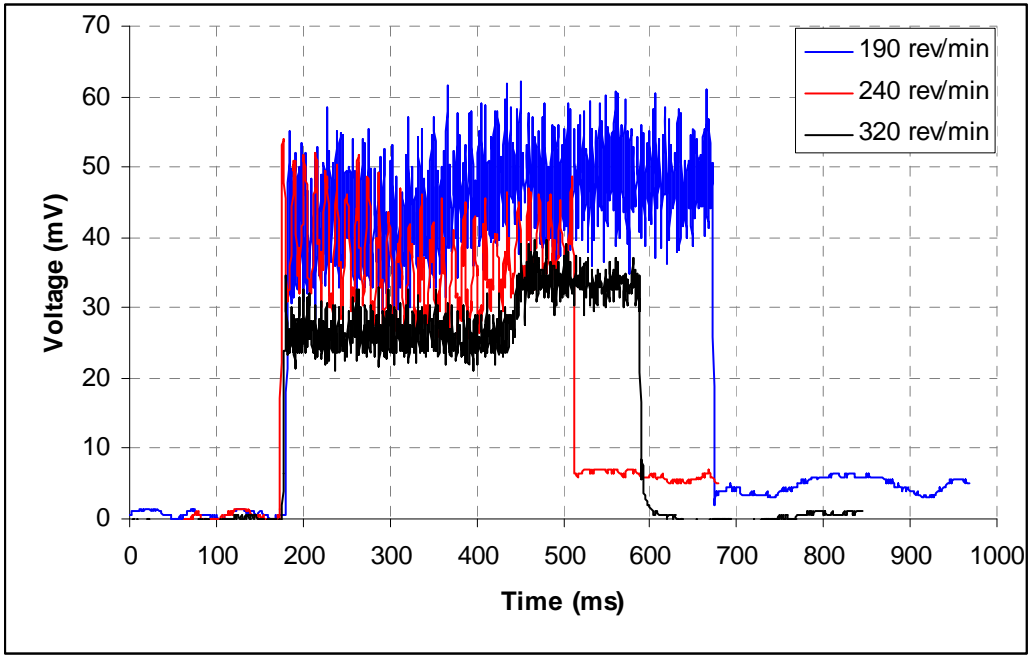

(b)

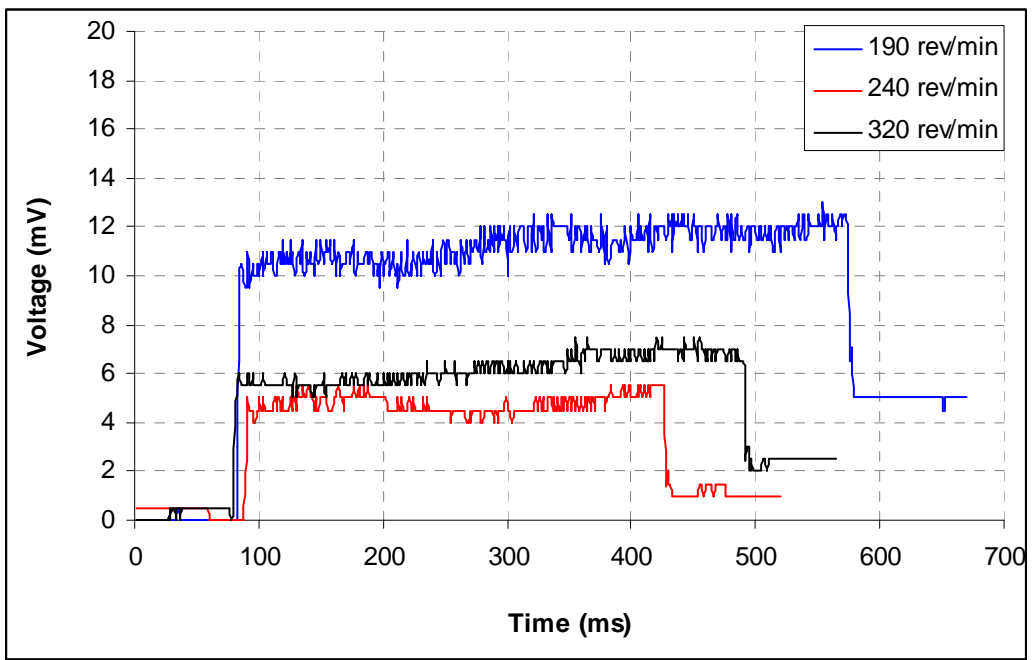

(c) 
Figure 7. Comparison of cutting forces at different rotational speeds at a feed speed of $0.081 \mathrm{~mm} \mathrm{rev}^{-1}$ for CrN-coated cutting tools: (a) cutting force: $y=-0.501 x+1.0698$ $\left(R^{2}=0.9998 ; P<0.001\right)$, (b) feed force: $y=80.26 x+124.9\left(R^{2}=0.9995 ; P<0.001\right)$ and (c) radial force: $y=7.94 x+128.5\left(R^{2}=0.9993 ; P<0.001\right) . y$ and $x$ values are in $\mathrm{kN}$ and in $\mathrm{mV}$, respectively.

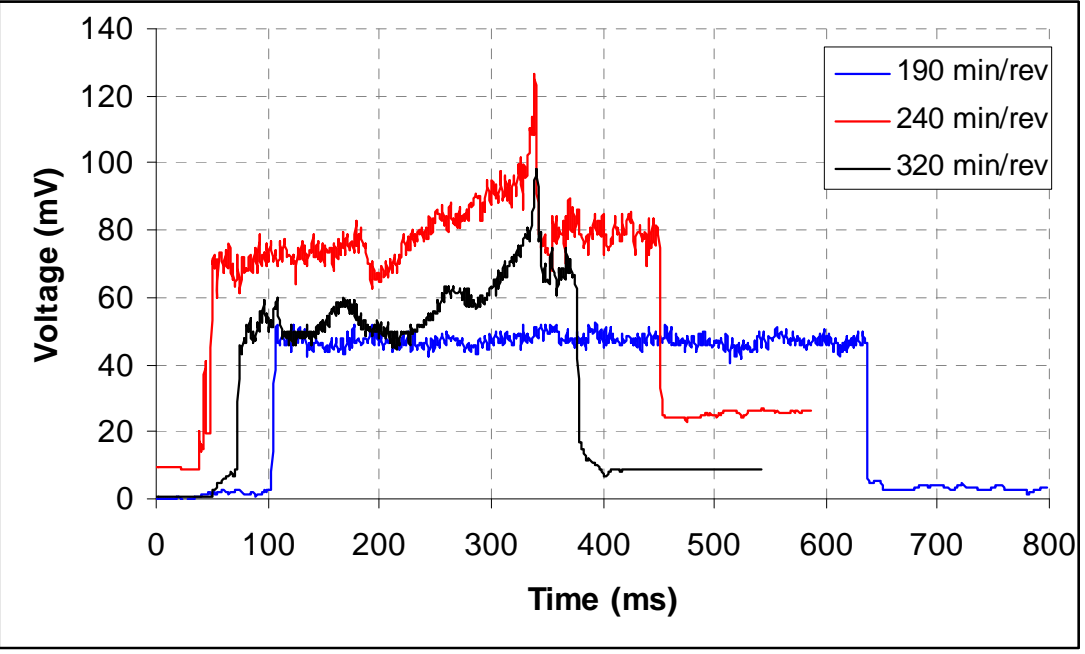

(a)

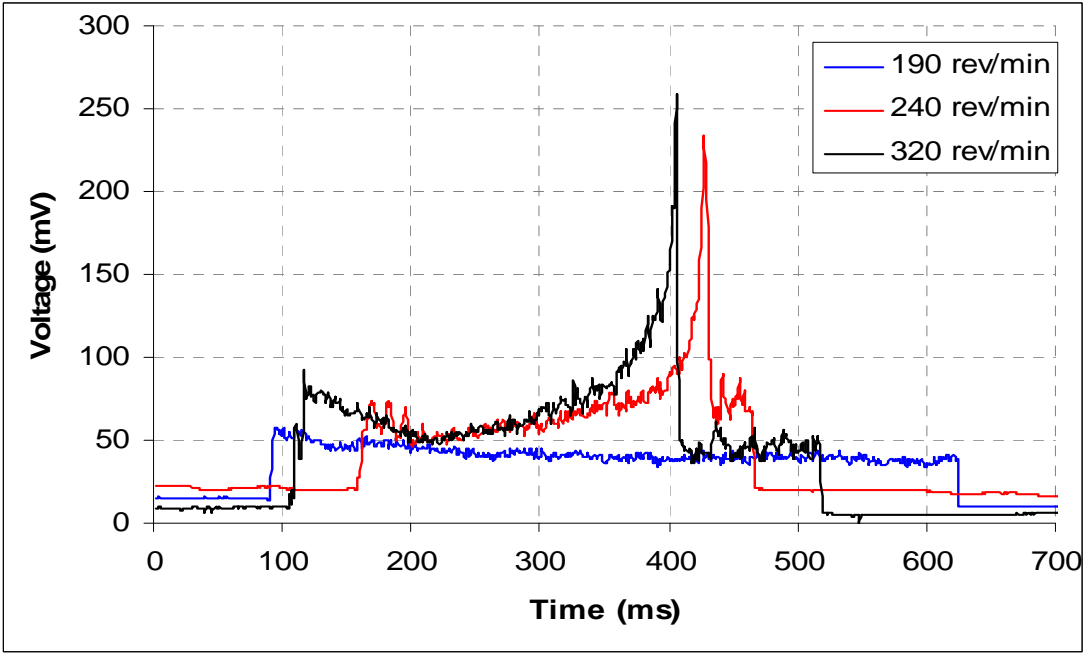

(b)

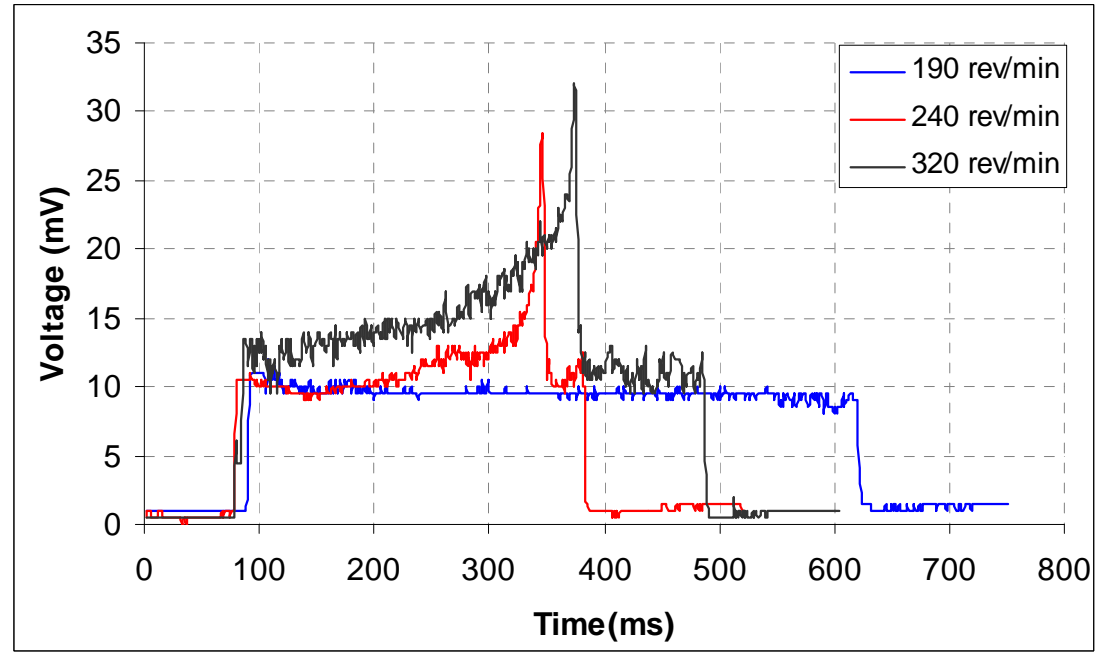

(c) 


\subsection{Microstructural Aspects}

SEM Line Scan analysis of cross-section of prenitrided high carbon steel coated with TiN and CrN is shown in Figs. 8 and 9, respectively.

Figure 8. EDS line-scan analysis of TiN-coated sample.
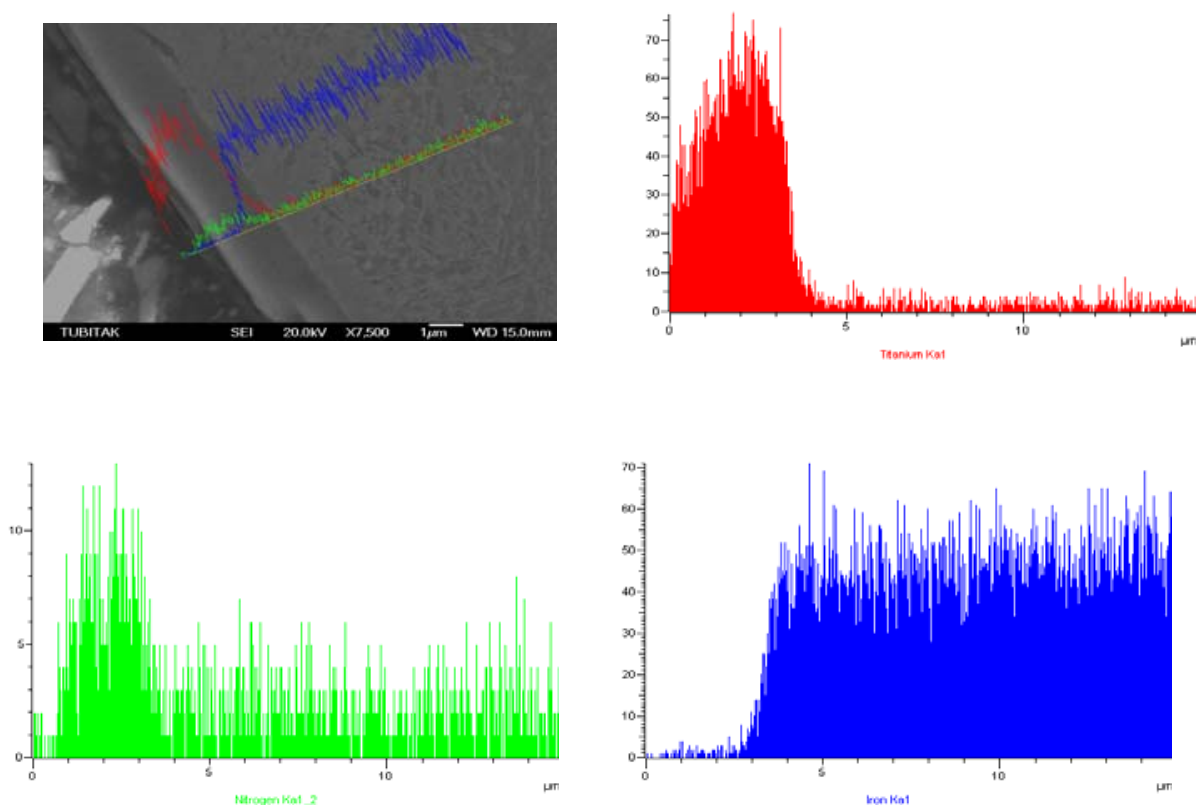

Variation in the contents of Ti, nitrogen $(\mathrm{N})$ and iron $(\mathrm{Fe})$ across the coated region is given in Fig. 8, and variation in those of $\mathrm{Cr}, \mathrm{N}$ and $\mathrm{Fe}$ is given in Fig. 9, starting from the cladding layer towards the substrate, determined using the line scan analysis technique.

Figure 9. EDS line-scan analysis of $\mathrm{CrN}$-coated sample.
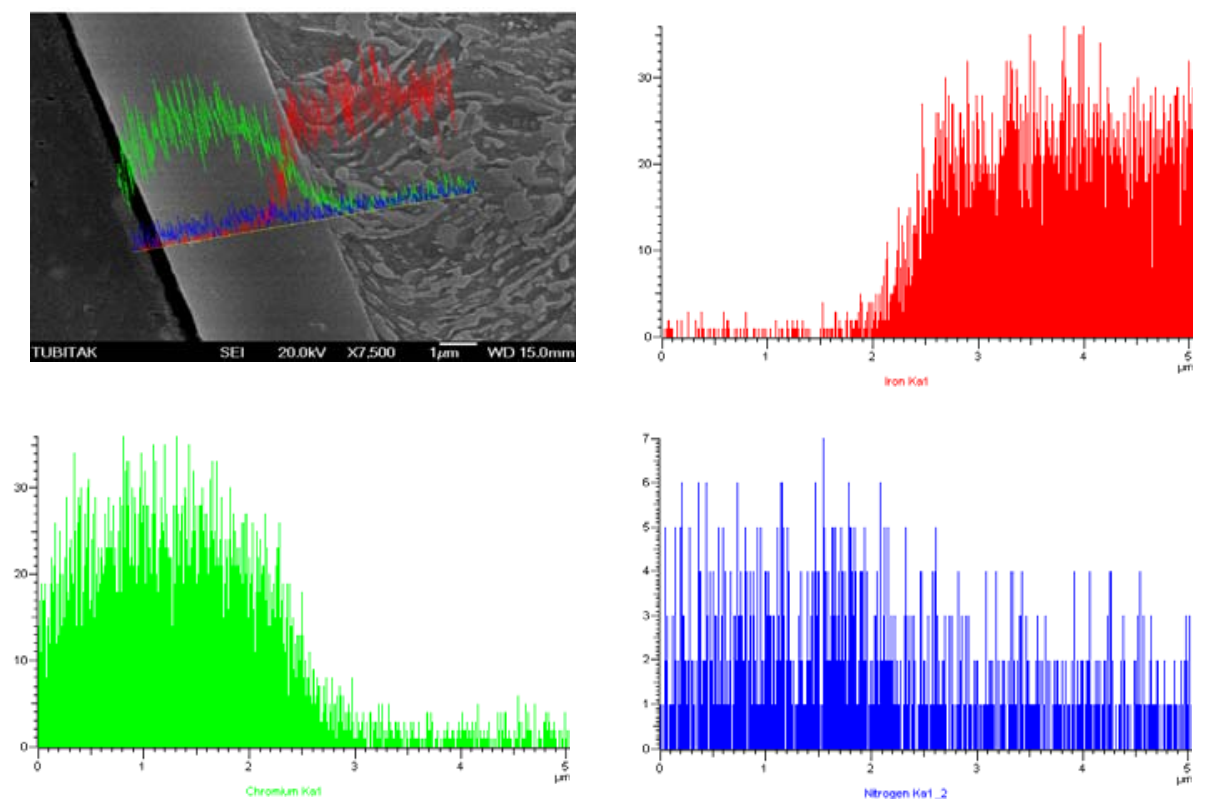
As seen in Fig. 8, Ti is present substantially in the outer region of the cladding layer, and its presence decreases sharply on the sub-surface line between the white layer and the cladding layer. Hardly any $\mathrm{Ti}$ exists within the matrix. It was observed that $\mathrm{N}$ heavily existed in the prenitrided surface. However, it was also observed that $\mathrm{N}$ existed in an evenly diffused way in the white layer and within the matrix itself. A small amount of Fe seemed to diffuse to the cladding region. Starting from the white layer, its ratio increased rapidly towards the substrate (Fig. 8). The reason for the sharp increase in the amounts of $\mathrm{Ti}$ and $\mathrm{N}$ from the outer to the inner region can be attributed to the relatively low temperature $\left(180{ }^{\circ} \mathrm{C}\right)$ used, and the short period of the treatment time.

Fig. 10 presents the XRD spectrum of cross-section of prenitrided high carbon steel coated with TiN. From the diffraction peaks of cross-section, $\mathrm{Ti}_{2} \mathrm{~N}, \mathrm{TiN}, \alpha-\mathrm{Fe}$, and $\gamma^{\prime}-\mathrm{Fe}_{4} \mathrm{~N}$ phases are present in the coated layer of TiN-coated specimen. Fig. 11 exhibits X-ray diffraction spectrum of cross-section of prenitrided high carbon steel coated with $\mathrm{CrN}$. It can be seen that $\mathrm{CrN}, \alpha-\mathrm{Fe}$, and $\gamma^{\prime}-\mathrm{Fe}_{4} \mathrm{~N}$ phases are present in the coated layer of CrN-coated specimen.

Figure 10. TiN-coated specimen: (a) SEM micrograph showing the microstructure across the cross-section and (b) XRD spectrum.

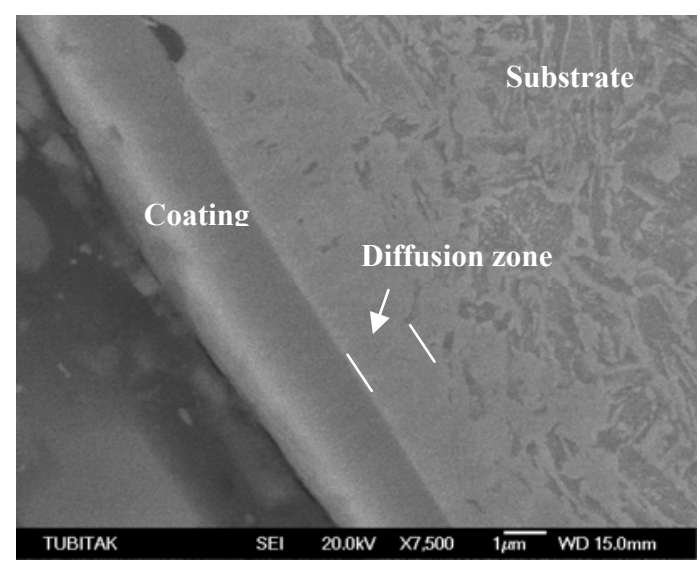

(a)

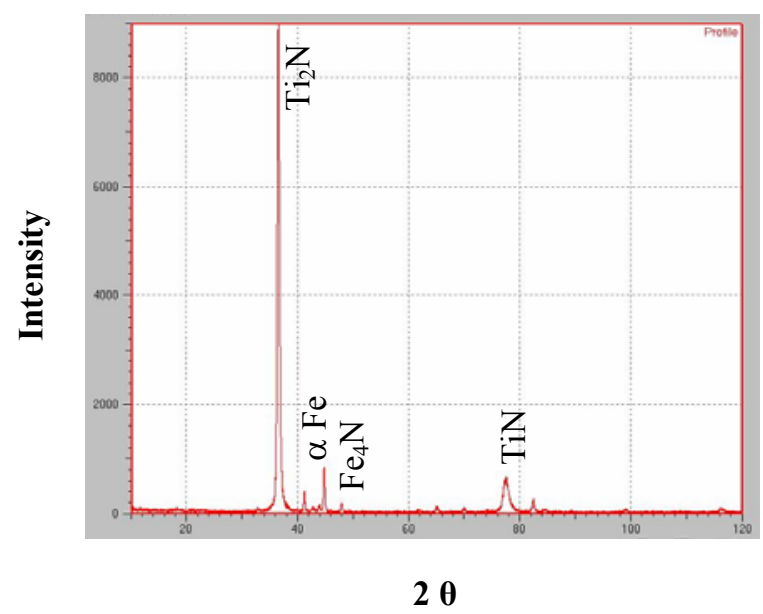

(b) 
Figure 11. CrN-coated specimen: (a) SEM micrograph showing the microstructure across the cross-section and (b) XRD spectrum.

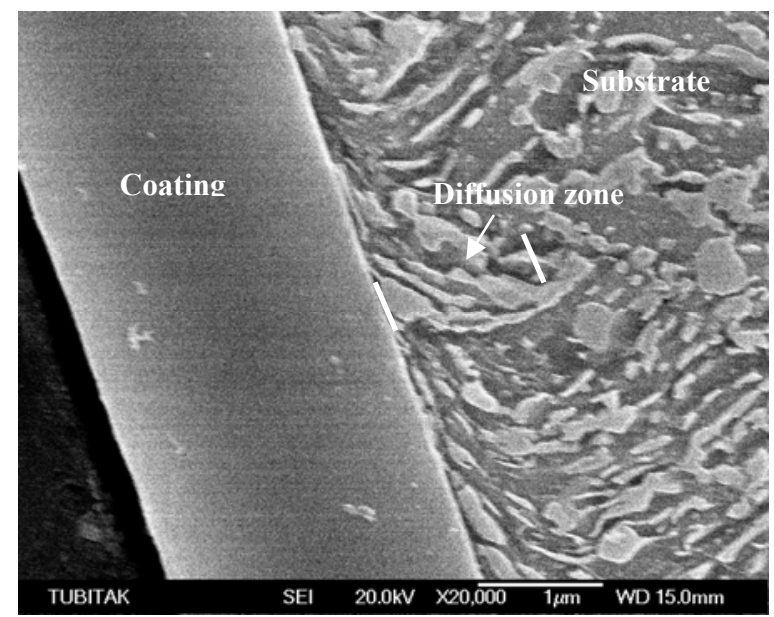

(a)

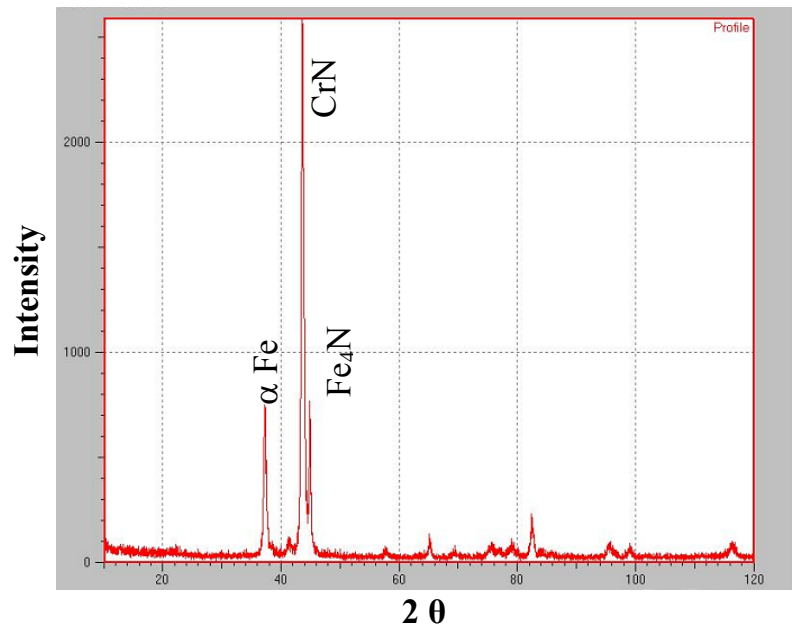

(b)

\section{Conclusions}

As metal cutting process is quite complicated, it is very difficult to determine mechanisms in which cutting parameters affect tool wear and cutting forces. We used an optoelectronic sensor system to monitor the tool wear without interrupting machining process. We based the provision of a reliable and sensitive technique for on-line monitoring of the tool wear on the basic idea of measuring the flank wear and notching on the tool indirectly by correlating it with the workpiece dimension. In the present work, thorough experimental investigations were conducted in order to study the effects of prenitrided substrate and surface mechanical treatments on the coating adhesion and cutting performance of TiNand $\mathrm{CrN}$-coated cutting tools. Accordingly, appropriate turning experiments were conducted at different cutting conditions to determine the amount of tool wear taking place on the flank.

Our experimental results revealed that prenitrided substrate and surface treatment enhanced coating adhesion properties and led to an increased turning performance of cutting inserts in the case of TiN- 
coating. This is also verified by observed changes in the cutting forces during the cutting process. The substrate on CrN plating worsened the adhesion strength, as well as the tool cutting performance.

\section{Acknowledgments}

The authors gratefully acknowledge financial support from the Scientific Research Projects Units of University of Firat (FÜBAP Project No: 1116) and of Abant Izzet Baysal University.

\section{References}

1. Hogmark, S.; Jacobson, S.; Larsson, M. Design and evaluation of tribological coatings. Wear 2000, 246, 20-33.

2. Höck, K.; Spies H.J.; Larisch, B.; Leonhardt, G.; Buecken, B. Wear resistance of prenitrided hard coated steels for tools and machine components. Surface and Coatings Technology 1996, 88, 4449.

3. Kickel, J.; Suhaib, A.N.; Yilbas, B.S.; Nizam, S.M. Evaluation of the wear of plasma-nitrided and TiN-coated HSS drills using conventional and Micro-PIXE techniques. Wear 2000, 239, 155 167.

4. Ürgen, M.; Çakır, A. The effect of heating on corrosion behavior of TiN- and CrN-coated steels. Surface and Coatings Technology 1997, 96, 236-244.

5. Fox-Robinovich, G.S.; Kovalev, A.I.; Afanasyev, S.N. Characteristic features of wear in tools made of high-speed steels with surface engineered coatings I. Wear characteristics of surface engineered high-speed steel cutting tools. Wear 1996, 201, 38-44.

6. Bell, T.; Mao, K.; Sun, Y. Surface engineering design: modelling surface engineering systems for improved tribological performance. Surface and Coating Technology 1998, 108-109, 360-368.

7. Quaeyhaegens, C.; Kerkhofs, M.; Stals, L.M.; Stappen, M.V. Promising developments for new applications. Surface and Coating Technology 1996, 80, 181-184.

8. Lii, D.F. Wear behaviour of TiAlN films deposited on plasma nitrided high speed steel. Surface Engineering 1998, 14 (4), 305-308.

9. Sato, T.; Sugai, K.; Ueda, S.; Matsunami, K.; Yasuoka, M. Development of a hybrid coating process as an advanced surface modification for cutting tools and moulds. Surface and Coatings Technology 2003, 169-170, 45-48.

10. Narojczyk, J.; Werner, Z.; Piekoszewski, J.; Szymczyk, W. Effects of nitrogen implantation on lifetime of cutting tools made of SK5M tool steel. Vacuum 2005, 78, 229-223.

11. Zlatanovic, M.; Popovic, N.; Bogdanov, Z. Formation of magnetite overlayer on nitrocarburized low-alloy steel by plasma postoxidation. Surface and Coatings Technology 2004, 188-189, 307313.

12. Govekar, E.; Gradisek, J.; Grabec, I. Analysis of acoustic emission signals and monitoring of machining processes. Ultrasonic 2000, 38, 598-603.

13. Chi, L.A.; Domfield, D.A. A self-organizing approach to the prediction and detection of tool wear. ISA Transactions 1998, 37, 239-255.

14. Li, X. A brief review: acoustic emission method for tool wear monitoring during turning. International Journal of Machine Tools and Manufacture 2002, 42, 157-165. 
15. Li, X.; Djordjevich, A.; Venuvinod, P.K. Current-sensor-based feed cutting force intelligent estimation and tool wear condition monitoring. IEEE Transactions on Industrial Electronics 2000, 47(3), 697-702.

16. Smith, G.C.; Lee, S.S. A method for detecting tool wear on a CNC lathe using a doppler radar detector. The International Journal of Advanced Manufacturing Technology 2005, 25, 270-280.

17. Cakan, A.; Investigation of Mechanical Properties, Microstructures and Cutting Performances of Cutting Tools Produced from Surface Hardened and Coated AISI 1070 Steel, PhD Thesis, Firat University, Graduate School of Natural and Applied Sciences, Department of Metallurgy Education, Elazig, Turkey, 2006, pp. 125.

18. Choudhury, S.K.; Jain, V.K.; Rama Rao, Ch.V.V. On-line monitoring of tool wear in turning using a neural network. International Journal of Machine Tools and Manufacture 1999, 39, 489504.

19. Sick, B. Fusion of hard and soft computing techniques in indirect online tool wear monitoring. IEEE Transaction on Systems, Man and Cybernetics 2002, 32(2), 80-91.

20. Yaldız, S.; Ünsaçar, F. A dynamometer design for measurement the cutting forces on turning. Measurement 2006, 39, 80-89.

21. Karabay, S. Design criteria for electro-mechanical transducers and arrangement for measurement of strains due to metal cutting forces acting on dynamometers. Materials and Design 2007, 28(2), 496-506.

22. Yaldız, S.; Ünsaçar, F.; Sağlam, H.; Işık, H. Design, development and testing of a four-component milling dynamometer for the measurement of cutting force and torque. Mechanical Systems and Signal Processing 2007, 21(3), 1499-1511.

23. Liu, Q.; Altintas, Y. On-line monitoring of flank wear in turning with multilayered feed-forward neural network. International Journal of Machine Tools and Manufacture 1999, 39, 1945-1959.

24. Su, Y.L.; Liu, T.H.; Su, C.T.; Yur, J.P.; Kao, W.H.; Yao, S.H. Tribological characteristics and cutting performance of $\mathrm{Cr}_{\mathrm{x} \%} \mathrm{C}$-coated carbide tools. Journal of Materials Processing Technology 2004, 153-154, 699-706.

25. Su, Y.L.; Liu, T.H.; Su, C.T.; Yao, S.H.; Kao, W.H.; Cheng, K.W. Wear of CrC-coated carbide tools in dry machining. Journal of Materials Processing Technology 2006, 171, 108-117.

26. Scheerer, H.; Hoche, T. H.; Broszeit, E.; Schramm, B.; Abele, E.; Berger, C. Effects of the chromium to aluminum content on the tribology in dry machining using $(\mathrm{Cr}, \mathrm{Al}) \mathrm{N}$ coated tools. Surface and Coatings Technology 2005, 200, 203- 207.

(C) 2008 by the authors; licensee Molecular Diversity Preservation International, Basel, Switzerland. This article is an open-access article distributed under the terms and conditions of the Creative Commons Attribution license (http://creativecommons.org/licenses/by/3.0/). 